\title{
EmoNet: Fine-Grained Emotion Detection with Gated Recurrent Neural Networks
}

\author{
Muhammad Abdul-Mageed \\ School of Library, Archival \& \\ Information Studies \\ University of British Columbia \\ muhammad.mageed@ubc.ca
}

\author{
Lyle Ungar \\ Computer and Information Science \\ University of Pennsylvania \\ ungaracis. upenn. edu
}

\begin{abstract}
Accurate detection of emotion from natural language has applications ranging from building emotional chatbots to better understanding individuals and their lives. However, progress on emotion detection has been hampered by the absence of large labeled datasets. In this work, we build a very large dataset for fine-grained emotions and develop deep learning models on it. We achieve a new state-of-the-art on 24 fine-grained types of emotions (with an average accuracy of $87.58 \%$ ). We also extend the task beyond emotion types to model Robert Plutchik's 8 primary emotion dimensions, acquiring a superior accuracy of $95.68 \%$.
\end{abstract}

\section{Introduction}

According to the Oxford English Dictionary, emotion is defined as "[a] strong feeling deriving from one's circumstances, mood, or relationships with others." ${ }^{1}$ This "standard" definition identifies emotions as constructs involving something innate that is often invoked in social interactions and that aids in communicating with others(Hwang and Matsumoto, 2016). It is no exaggeration that humans are emotional beings: Emotions are an integral part of human life, and affect our decision making as well as our mental and physical health. As such, developing emotion detection models is important; they have a wide array of applications, ranging from building nuanced virtual assistants that cater for the emotions of their users to detecting the emotions of social media users in order to understand their mental and/or physical health.

\footnotetext{
${ }^{1}$ https://en.oxforddictionaries.com/ definition/emotion.
}

However, emotion detection has remained a challenging task, partly due to the limited availability of labeled data and partly due the controversial nature of what emotions themselves are (Aaron C. Weidman and Tracy, 2017).

Recent advances in machine learning for natural language processing (NLP) suggest that, given enough labeled data, there should be an opportunity to build better emotion detection models. Manual labeling of data, however, is costly and so it is desirable to develop labeled emotion data without annotators. While the proliferation of social media has made it possible for us to acquire large datasets with implicit labels in the form of hashtags (Mohammad and Kiritchenko, 2015), such labels are noisy and reliable.

In this work, we seek to enable deep learning by creating a large dataset of fine-grained emotions using Twitter data. More specifically, we harness cues in Twitter data in the form of emotion hashtags as a way to build a labeled emotion dataset that we then exploit using distant supervision (Mintz et al., 2009) (the use of hashtags as a surrogate for annotator-generated emotion labels) to build emotion models grounded in psychology. We construct such a dataset and exploit it using powerful deep learning methods to build accurate, high coverage models for emotion prediction. Overall, we make the following contributions: 1) Grounded in psychological theory of emotions, we build a large-scale, high quality dataset of tweets labeled with emotions. Key to this are methods to ensure data quality, 2) we validate the data collection method using human annotations, 3) we develop powerful deep learning models using a gated recurrent network to exploit the data, yielding new state-of-the-art on 24 fine-grained types of emotions, and 4) we extend the task beyond these emotion types to model Plutick's 8 primary emotion dimensions. 
Our emotion modeling relies on distant supervision (Read, 2005; Mintz et al., 2009), the approach of using cues in data (e.g., hashtags or emoticons) as a proxy for "ground truth" labels as we explained above. Distant supervision has been investigated by a number of researchers for emotion detection (Tanaka et al., 2005; Mohammad, 2012; Purver and Battersby, 2012; Wang et al., 2012; Pak and Paroubek, 2010; Yang et al., 2007) and for other semantic tasks such as sentiment analysis (Read, 2005; Go et al., 2009) and sarcasm detection (González-Ibánez et al., 2011). In these works, authors successfully use emoticons and/or hashtags as marks to label data after performing varying degrees of data quality assurance. We take a similar approach, using a larger collection of tweets, richer emotion definitions, and stronger filtering for tweet quality.

The remainder of the paper is organized as follows: We first overview related literature in Section 2, describe our data collection in Section 3.1, and the annotation study we performed to validate our distant supervision method in Section 4. We then describe our methods in Section 5, provide results in Section 6, and conclude in Section 8.

\section{Related Work}

\subsection{Computational Treatment of Emotion}

The SemEval-2007 Affective Text task (Strapparava and Mihalcea, 2007) [SEM07] focused on classification of emotion and valence (i.e., positive and negative texts) in news headlines. A total of 1,250 headlines were manually labeled with the 6 basic emotions of Ekman (Ekman, 1972) and made available to participants. Similarly, (Aman and Szpakowicz, 2007) describe an emotion annotation task of identifying emotion category, emotion intensity and the words/phrases that indicate emotion in blog post data of 4,090 sentences and a system exploiting the data. Our work differs from both that of SEM07 (Strapparava and Mihalcea, 2007) and (Aman and Szpakowicz, 2007) in that we focus on a different genre (i.e., Twitter) and investigate distant supervision as a way to acquire a significantly larger labeled dataset.

Our work is similar to (Mohammad, 2012; Mohammad and Kiritchenko, 2015), (Wang et al., 2012), and (Volkova and Bachrach, 2016) who use distant supervision to acquire Twitter data with emotion hashtags and report analyses and experiments to validate the utility of this approach. For example, (Mohammad, 2012) shows that by using a simple domain adaptation method to train a classifier on their data they are able to improve both precision and recall on the SemEval-2007 (Strapparava and Mihalcea, 2007) dataset. As the author points out, this is another premise that the selflabeled hashtags acquired from Twitter are consistent, to some degree, with the emotion labels given by the trained human judges who labeled the SemEval-2007 data. As pointed out earlier, (Wang et al., 2012) randomly sample a set of 400 tweets from their data and human-label as relevant/irrelevant, as a way to verify the distant supervision approach with the quality assurance heuristics they employ. The authors found that the precision on a test set is $93.16 \%$, thus confirming the utility of the heuristics. (Wang et al., 2012) provide a number of important observations, as conclusions based on their work. These include that since they are provided by the tweets' writers, the emotion hashtags are more natural and reliable than the emotion labels traditionally assigned by annotators to data by a few annotators. This is the case since in the lab-condition method annotators need to infer the writers emotions from text, which may not be accurate. Additionally, (Volkova and Bachrach, 2016) follow the same distant supervision approach and find correlations of users' emotional tone and the perceived demographics of these users' social networks exploiting the emotion hashtag-labeled data. Our dataset is more than an order of magnitude larger than (Mohammad, 2012) and (Volkova and Bachrach, 2016) and the range of emotions we target is much more fine grained than (Mohammad, 2012; Wang et al., 2012; Volkova and Bachrach, 2016) since we model 24 emotion types, rather than focus on $\leq 7$ basic emotions.

(Yan et al., 2016; Yan and Turtle, 2016a,b) develop a dataset of 15,553 tweets labeled with 28 emotion types and so target a fine-grained range as we do. The authors instruct human annotators under lab conditions to assign any emotion they feel is expressed in the data, allowing them to assign more than one emotion to a given tweet. A set of 28 chosen emotions was then decided upon and further annotations were performed using Amazon Mechanical Turk (AMT). The authors cite an agreement of 0.50 Krippendorff's alpha $(\alpha)$ between the lab/expert annotators, and an $(\alpha)$ of 0.28 between experts and AMT workers. EmoTweet- 
28 is a useful resource. However, the agreement between annotators is not high and the set of assigned labels do not adhere to a specific theory of emotion. We use a much larger dataset and report an accuracy of the hashtag approach at $90 \%$ based on human judgement as reported in Section 4.

\subsection{Mood}

A number of studies have also been performed to analyze and/or model mood in social media data. (De Choudhury et al., 2012) identify more than 200 moods frequent on Twitter as extracted from psychological literature and filtered by AMT workers. They then collect tweets which have one of the moods in their mood lexicon in the form of a hashtag. To verify the quality of the mood data, the authors run AMT studies where they ask workers whether a tweet displayed the respective mood hashtag or not and find that in $83 \%$ of the cases hashtagged moods at the end of posts did capture users' moods, whereas for posts with mood hashtags anywhere in the tweet, only $58 \%$ of the cases capture the mood of users. Although they did not build models for mood detection, the annotation studies (De Choudhury et al., 2012) perform further support our specific use of hashtags to label emotions. (Mishne and De Rijke, 2006) collect user-labeled mood from blog post text on LiveJournal and exploit them for predicting the intensity of moods over a time span rather than at the post level. Similarly, (Nguyen, 2010) builds models to infer patterns of moods in a large collection of LiveJournal posts. Some of the moods in these LiveJournal studies (e.g., hungry, cold), as (De Choudhury et al., 2012) explain, would not fit any psychological theory. Our work is different in that it is situated in psychological theory of emotion.

\subsection{Deep Learning for NLP}

In spite of the effectiveness of feature engineering for NLP, it is a labor intensive task that also needs domain expertise. More importantly, feature engineering falls short of extracting and organizing all the discriminative information from data (LeCun et al., 2015; Goodfellow et al., 2016). Neural networks (Goodfellow et al., 2016) have emerged as a successful class of methods that has the power of automatically discovering the representations needed for detection or classification and has been successfully applied to multiple NLP tasks. A line of studies in the literature (e.g., (Labutov and Lip- son, 2013; Maas et al., 2011; Tang et al., 2014b,a) aim to learn sentiment-specific word embeddings (Bengio et al., 2003; Mikolov et al., 2013) from neighboring text. Another thread of research focuses on learning semantic composition (Mitchell and Lapata, 2010), including extensions to phrases and sentences with recursive neural networks (a class of syntax-tree models) (Socher et al., 2013; Irsoy and Cardie, 2014; Li et al., 2015) and to documents with distributed representations of sentences and paragraphs (Le and Mikolov, 2014; Tang et al., 2015) for modeling sentiment.

Long-short term memory (LSTM) (Hochreiter and Schmidhuber, 1997) and Gated Recurrent Neural Nets (GRNNs) (Cho et al., 2014; Chung et al., 2015), variations of recurrent neural networks (RNNs), a type of networks suitable for handling time-series data like speech (Graves et al., 2013) or handwriting recognition (Graves, 2012; Graves and Schmidhuber, 2009), have also been used successfully for sentiment analysis (Ren et al., 2016; Liu et al., 2015; Tai et al., 2015; Tang et al., 2015; Zhang et al., 2016). Convolutional neural networks (CNNs) have also been quite successful in NLP, and have been applied to a range of sentence classification tasks, including sentiment analysis (Blunsom et al., 2014; Kim, 2014; Zhang et al., 2015). Other architectures have also been recently proposed (e.g., (Bradbury et al., 2016)). A review of neural network methods for NLP can be found in (Goldberg, 2016).

\section{Data}

\subsection{Collection of a Large-Scale Dataset}

To be able to use deep learning for modeling emotion, we needed a large dataset of labeled tweets. Since there is no such human-labeled dataset publicly available, we follow (Mohammad, 2012; Mintz et al., 2009; Purver and Battersby, 2012; González-Ibánez et al., 2011; Wang et al., 2012) in adopting distant supervision: We collect tweets with emotion-carrying hashtags as a surrogate for emotion labels. To be able to collect enough tweets to serve our need, we developed a list of hashtags representing each of the 24 emotions proposed by Robert Plutchick (Plutchik, 1980, 1985, 1994). Plutchik (Plutchik, 2001) organizes emotions in a three-dimensional circumplex model analogous to the colors on a color wheel. The cone's vertical dimension represents intensity, and the 3 circle represent degrees of similarity 
Figure 1: Plutchik's wheel of emotion.

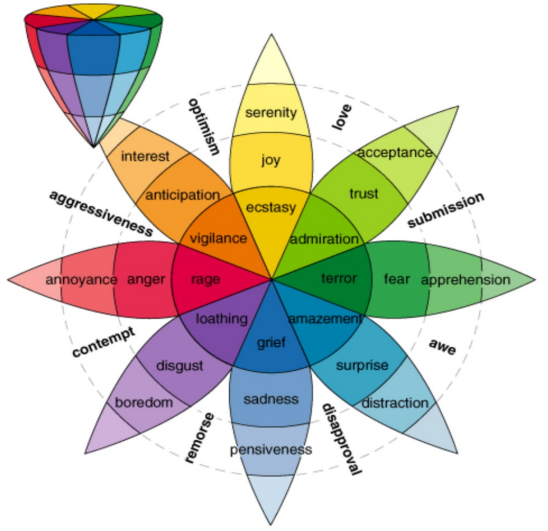

among the various emotion types. The eight sectors are meant to capture that there are eight primary emotion dimensions arranged as four pairs of opposites. Emotions in the blank spaces are the primary emotion dyads (i.e., emotions that are mixtures of two of the primary emotions). For this work, we exclude the dyads in the exploded model from our treatment. For simplicity, we refer to the circles as plutchik-1: with the emotions \{admiration, amazement, ecstasy, grief, loathing, rage, terror, vigilance $\},$ plutchik-2: with the emotions fjoy, trust, fear, surprise, sadness, disgust, anger, anticipation $\}$, and plutchik-3: with the emotions \{acceptance, annoyance, apprehension, boredom, distraction, interest, pensiveness, serenity\}. The wheel is shown in Figure 1.

For each emotion type, we prepared a seed set of hashtags representing the emotion. We used Google synonyms and other online dictionaries and thesauri (e.g., www.thesaurus. com) to expand the initial seed set of each emotion. We acquire a total of 665 emotion hashtags across the 24 emotion types. For example, for the joy emotion, a subset of the seeds in our expanded set is \{ "happy", "happiness", "joy", "joyful", "joyfully", "delighted", "feelingsunny", "blithe", "beatific", "exhilarated", "blissful", "walkingonair", "jubilant" $\}$. We then used the expanded set to extract tweets with hashtags from the set from a number of massive-scale in-house Twitter datasets. We also used Twitter API to crawl Twitter with hashtags from the expanded set. Using this method, we were able to acquire a dataset of about $1 / 4$ billion tweets covering an extended time span from July 2009 till January 2017.

\subsection{Preprocessing and Quality Assurance}

Twitter data are very noisy, not only because of use of non-standard typography (which is less of a problem here) but due to the many duplicate tweets and the fact that tweets often have multiple emotion hashtags. Since these reduce our ability to build accurate models, we need to clean the data and remove duplicates. Starting with $>1 / 4$ billion tweets, we employ a rigorous and strict pipeline. This results in a vastly smaller set of about 1.6 million dependable labeled tweets.

Since our goal is to create non-overlapping categories at the level of a tweet, we first removed all tweets with hashtags belonging to more than one emotion of the 24 emotion categories. Since it was observed (e.g., (Mohammad, 2012; Wang et al., 2012)) and also confirmed by our annotation study as described in Section 4, that hashtags in tweets with URLs are less likely to correlate with a true emotion label, we remove all tweets with URLs from our data. We filter out duplicates using a two-step procedure: 1) we remove all retweets (based on existence of the token "RT" regardless of case) and 2) we use the Python library pandas http://pandas. pydata.org/ "drop_duplicates" method to compare the tweet texts of all the tweets after normalizing character repetitions [all consecutive characters of $>2$ to 2] and user mentions (as detected by a string starting with an "@” sign). We then performed a manual inspection of a random sample of 1,000 tweets from the data and found no evidence of any remaining tweet duplicates.

Next, even though the emotion hashtags themselves are exclusively in English, we observe the data do have tweets in languages other than English. This is due to code-switching, but also to the fact that our data dates back to 2009 and Twitter did not allow use of hashtags for several non-English languages until 2012. To filter out non-English, we use the langid (Lui and Baldwin, 2012) (https://github.com/ saffsd/langid.py) library to assign language tags to the tweets. Since the common wisdom in the literature (e.g., (Mohammad, 2012; Wang et al., 2012)) is to restrict data to hashtags occurring in final position of a tweet, we investigate correlations between a tweet's relevance and emotion hashtag location in Section 4 and test models exclusively on data with hashtags occurring in final position. We also only use tweets con- 
taining at least 5 words.

Table 2 shows statistics of the data after applying our cleaning, filtering, language identification, and deduplication pipeline. Since our focus is on English, we only show statistics for tweets tagged with an "en" (for "English") label by langid. Table 2 provides three types of relevant statistics: 1) counts of all tweets, 2) counts of tweets with at least 5 words and the emotion hashtags occurring in the last quarter of the tweet text (based on character count), and 3) counts of tweets with at least 5 words and the emotion hashtags occurring as the final word in the tweet text. As the last column in Table 2 shows, employing our most strict criterion where an emotion hashtag must occur finally in a tweet of a minimal length 5 words, we acquire a total of 1,608,233 tweets: 205,125 tweets for plutchik-1, 790,059 for plutchik-2, and 613,049 for plutchik-3. ${ }^{2}$

\begin{tabular}{|l|l|l|l|}
\hline Emotion & ct & ct $@ \mathbf{l q}$ & ct $@$ end \\
\hline admiration & 292,153 & 150,509 & 112,694 \\
\hline amazement & 568,255 & 358,472 & 34,826 \\
\hline ecstasy & 54,174 & 34,307 & 23,856 \\
\hline grief & 102,980 & 33,141 & 12,568 \\
\hline loathing & 90,465 & 41,787 & 456 \\
\hline rage & 30,994 & 11,777 & 4,749 \\
\hline terror & 84,827 & 25,908 & 15,268 \\
\hline vigilance & 6,171 & 1,028 & 708 \\
\hline plutchik-1 & $\mathbf{1 , 2 3 0 , 0 1 9}$ & $\mathbf{6 5 6 , 9 2 9}$ & $\mathbf{2 0 5 , 1 2 5}$ \\
\hline \hline anger & 131,082 & 82,447 & 56,472 \\
\hline anticipation & 67,175 & 36,846 & 26,655 \\
\hline disgust & 212,770 & 145,052 & 52,067 \\
\hline fear & 302,989 & 153,513 & 98,657 \\
\hline joy & 974,226 & 522,689 & 330,738 \\
\hline sadness & $1,252,192$ & 762,901 & 142,300 \\
\hline surprise & 143,755 & 78,570 & 53,915 \\
\hline trust & 198,619 & 103,332 & 29,255 \\
\hline plutchik-2 & $\mathbf{3 , 2 8 2 , 8 0 8}$ & $\mathbf{1 , 8 8 5 , 3 5 0}$ & $\mathbf{7 9 0 , 0 5 9}$ \\
\hline \hline acceptance & 138,899 & 54,706 & 16,522 \\
\hline annoyance & 954,027 & 791,869 & 364,135 \\
\hline apprehension & 29,174 & 11,650 & 7,828 \\
\hline boredom & 872,246 & 583,994 & 152,105 \\
\hline distraction & 122,009 & 52,633 & 617 \\
\hline interest & 113,555 & 67,216 & 56,659 \\
\hline pensiveness & 11,751 & 5,012 & 3,513 \\
\hline serenity & 97,467 & 36,817 & 11,670 \\
\hline plutchik-3 & $\mathbf{2 , 3 3 9 , 1 2 8}$ & $\mathbf{1 , 6 0 3 , 8 9 7}$ & $\mathbf{6 1 3 , 0 4 9}$ \\
\hline \hline ALL & $\mathbf{6 , 8 5 1 , 9 5 5}$ & $\mathbf{4 , 1 4 6 , 1 7 6}$ & $\mathbf{1 , 6 0 8 , 2 3 3}$ \\
\hline & & & \\
\hline
\end{tabular}

Table 2: Data statistics.

\section{Annotation Study}

In their work, (Wang et al., 2012) manually label a random sample of 400 tweets extracted with hash-

\footnotetext{
${ }^{2}$ The data can be acquired by emailing the first author. The distribution is in the form of tweet ids and labels, to adhere to Twitter conditions.
}

tags in a similar way as we acquire our data and find that human annotators agree $93 \%$ of the time with the hashtag emotion type if the hashtag occurs as the last word in the tweet. We wanted to validate our use of hashtags in a similar fashion and on a bigger random sample. We had human annotators label a random sample of 5,600 tweets that satisfy our preprocessing pipeline. Manual inspection during annotation resulted in further removing a negligible 16 tweets that were found to have problems. For each of the remaining 5,584 tweets, the annotators assign a binary tag from the set $\{$ relevant, irrelevant $\}$ to indicate whether a tweet carries an emotion category as assigned using our distant supervision method or not. Annotators assigned $61.37 \%(n=3,427)$ "relevant" tags and $38.63 \%(n=2,157)$ "irrelevant" tags. Our analysis of this manually labeled dataset also supports the findings of (Wang et al., 2012): When we limit position of the emotion hashtag to the end of a tweet, we acquire $90.57 \%$ relevant data. We also find that if we relax the constraint on the hashtag position such that we allow the hashtag to occur in the last quarter of a tweet (based on a total tweet character count), we acquire $85.43 \%$ relevant tweets. We also find that only $23.20 \%$ ( $n=795$ out of 3,427 ) of the emotion carrying tweets have the emotion hashtags occurring in final position, whereas $31.75 \%$ ( $n=1,088$ out of 3,427 ) of the tweets have the emotion hashtags in the last quarter of the tweet string. This shows how enforcing a final hashtag location results in loss of a considerable number of emotion tweets. As shown in Table 2, only 1, 608, 233 tweets out of a total of $6,851,955$ tweets $(\%=23,47)$ in our bigger dataset have emotion hashtags occurring in final position. Overall, we agree with (Mohammad, 2012; Wang et al., 2012) that the accuracy acquired by enforcing a strict pipeline and limiting to emotion hashtags to final position is a reasonable measure for warranting good-quality data for training supervised systems, an assumption we have also validated with our empirical findings here.

One advantage of using distant supervision under these conditions for labeling emotion data, as (Wang et al., 2012) also notes, is that the label is assigned by the writer of the tweet himself/herself rather than an annotator who could wrongly decide what category a tweet is. After all, emotion is a fuzzy concept and $>90 \%$ agreement as we 
report here is higher than the human agreement usually acquired on many NLP tasks. Another advantage of this method is obviously that it enables us to acquire a sufficiently large training set to use deep learning. We now turn to describing our deep learning methods.

\section{Methods}

For our core modeling, we use Gated Recurrent Neural Networks (GRNNs), a modern variation of recurrent neural networks (RNNs), which we now turn to introduce. For notation, we denote scalars with italic lowercase (e.g., $x$ ), vectors with bold lowercase (e.g.,x), and matrices with bold uppercase (e.g.,W).

Recurrent Neural Network A recurrent neural network (RNN) is one type of neural network architecture that is particularly suited for modeling sequential information. At each time step $t$, an RNN takes an input vector $\mathbf{x}_{t} \in \mathbb{R}^{n}$ and a hidden state vector $\mathbf{h}_{t-1} \in \mathbb{R}^{m}$ and produces the next hidden state $\mathbf{h}_{t}$ by applying the recursive operation:

$$
\mathbf{h}_{t}=f\left(\mathbf{W} \mathbf{x}_{t}+\mathbf{U} \mathbf{h}_{t-1}+\mathbf{b}\right)
$$

Where the input to hidden matrix $\mathbf{W} \in \mathbb{R}^{m \times n}$, the hidden to hidden matrix $\mathbf{U} \in \mathbb{R}^{m \times m}$, and the bias vector $\mathbf{b} \in \mathbb{R}^{m}$ are parameters of an affine transformation and $f$ is an element-wise nonlinearity. While an RNN can in theory summarize all historical information up to time step $\mathbf{h}_{t}$, in practice it runs into the problem of vanishing/exploding gradients (Bengio et al., 1994; Pascanu et al., 2013) while attempting to learn longrange dependencies.

LSTM Long short-term memory (LSTM) networks (Hochreiter and Schmidhuber, 1997) addresses this exact problem of learning long-term dependencies by augmenting an RNN with a memory cell $\mathbf{c}_{t} \in \mathbb{R}^{n}$ at each time step. As such, in addition to the input vector $\mathbf{x}_{t}$, the hiddent vector $\mathbf{h}_{t-1}$, an LSTM takes a cell state vector $\mathbf{c}_{t-1}$ and produces $\mathbf{h}_{t}$ and $\mathbf{c}_{t}$ via the following calculations:

$$
\begin{aligned}
\mathbf{i}_{t} & =\sigma\left(\mathbf{W}^{i} x_{t}+\mathbf{U}^{i} \mathbf{h}_{t-1}+\mathbf{b}^{i}\right) \\
\mathbf{f}_{t} & =\sigma\left(\mathbf{W}^{f} x_{t}+\mathbf{U}^{f} \mathbf{h}_{t-1}+\mathbf{b}^{f}\right) \\
\mathbf{o}_{t} & =\sigma\left(\mathbf{W}^{o} x_{t}+\mathbf{U}^{o} \mathbf{h}_{t-1}+\mathbf{b}^{o}\right) \\
\mathbf{g}_{t} & =\tanh \left(\mathbf{W}^{g} x_{t}+\mathbf{U}^{g} \mathbf{h}_{t-1}+\mathbf{b}^{g}\right) \\
\mathbf{c}_{t} & =\mathbf{f}_{t} \odot \mathbf{c}_{t-1}+\mathbf{i}_{t} \odot \mathbf{g}_{t} \\
\mathbf{h}_{t} & =\mathbf{o}_{t} \odot \tanh \left(\mathbf{c}_{t}\right)
\end{aligned}
$$

Where $\sigma(\cdot)$ and $\tanh (\cdot)$ are the element-wise sigmoid and hyperbolic tangent functions, $\odot$ the element-wise multiplication operator, and $\mathbf{i}_{t}, \mathbf{f}_{t}, \mathbf{o}_{t}$ are the input, forget, and output gates. The $\mathbf{g}_{t}$ is a new memory cell vector with candidates that could be added to the state. The LSTM parameters $\mathbf{W}_{j}$, $\mathbf{U}_{j}$, and $\mathbf{b}_{j}$ are for $j \epsilon\{i, f, o, g\}$.

GRNNs (Cho et al., 2014; Chung et al., 2015) propose a variation of LSTM with a reset gate $\mathbf{r}_{t}$, an update state $\mathbf{z}_{t}$, and a new simpler hidden unit $\mathbf{h}_{t}$, as follows:

$$
\begin{aligned}
\mathbf{r}_{t} & =\sigma\left(\mathbf{W}^{r} x_{t}+\mathbf{U}^{r} \mathbf{h}_{t-1}+\mathbf{b}^{r}\right) \\
\mathbf{z}_{t} & =\sigma\left(\mathbf{W}^{z} x_{t}+\mathbf{U}^{z} \mathbf{h}_{t-1}+\mathbf{b}^{z}\right) \\
\tilde{\mathbf{h}}_{t} & =\tanh \left(\mathbf{W} x_{t}+\mathbf{r}_{t} * \mathbf{U}^{\tilde{h}} \mathbf{h}_{t-1}+\mathbf{b}^{\tilde{h}}\right) \\
\mathbf{h}_{t} & =\mathbf{z}_{t} * \mathbf{h}_{t-1}+\left(1-\mathbf{z}_{t}\right) * \tilde{\mathbf{h}}_{t}
\end{aligned}
$$

The GRNN parameters $\mathbf{W}_{j}, \mathbf{U}_{j}$, and $\mathbf{b}_{j}$ are for $j \epsilon$ $\{r, z, \tilde{h}\}$. In this set up, the hidden state is forced to ignore a previous hidden state when the reset gate is close to 0 , thus enabling the network to forget or drop irrelevant information. Additionally, the update gate controls how much information carries over from a previous hidden state to the current hidden state (similar to an LSTM memory cell). We use GRNNs as they are simpler and faster than LSTM. For GRNNs, we use Theano (Theano Development Team, 2016).

Online Classifiers We compare the performance of the GRNNs to four online classifiers that are capable of handling the data size: Stochastic Gradient Descent (SGD), Multinomial Naive Bayes (MNB), Perceptron, and the Passive Agressive Classifier (PAC). These classifiers learn online from mini-batches of data. We use minibatches of 10,000 instances with all the four classifiers. We use the scikit-learn implementation of these classifiers (http: //scikit-learn. org).

Settings We aim to model Plutchik's 24 finegrained emotions as well as his 8 primary emotion dimensions where each 3 related types of emotion (perceived as varying in intensity) are combined in one dimension. We now turn to describing our experiments experiments.

\section{Experiments}

\subsection{Predicting Fine-Grained Emotions}

As explained earlier, Plutchik organizes the 24 emotion types in the 3 main circles that we will refer to as plutchik-1, plutchik-2, and plutchik-3. 


\begin{tabular}{|l|l|l|l|l|l|l|l|l|l|l|l|l|}
\hline Emotion & \multicolumn{2}{|l|}{ Qadir (2013) } & \multicolumn{2}{l|}{ Roberts (2012) } & \multicolumn{2}{l|}{ MD (2015) } & \multicolumn{2}{l|}{ Wang (2012) } & \multicolumn{2}{l|}{ Volkova (2016) } & \multicolumn{2}{l|}{ This work } \\
\hline anger & 400 & 0.44 & 583 & 0.64 & 1,555 & 0.28 & 457,972 & 0.72 & 4,963 & 0.80 & 56,472 & 0.75 \\
\hline anticip & - & - & - & - & - & - & - & - & - & - & 26,655 & 0.70 \\
\hline disgust & - & - & 922 & 0.67 & 761 & 0.19 & - & - & 12,948 & 0.92 & 52,067 & 0.82 \\
\hline fear & 592 & 0.54 & 222 & 0.74 & 2,816 & 0.51 & 11,156 & 0.44 & 9,097 & 0.77 & 98,657 & 0.74 \\
\hline joy & 1,005 & 0.59 & 716 & 0.68 & 8,240 & 0.62 & 567,487 & 0.72 & 15,559 & 0.79 & 330,738 & 0.91 \\
\hline sadness & 560 & 0.46 & 493 & 0.69 & 3,830 & 0.39 & 489,831 & 0.65 & 4,232 & 0.62 & 142,300 & 0.73 \\
\hline surprise & - & - & 324 & 0.61 & 3849 & 0.45 & 1,991 & 0.14 & 8,244 & 0.64 & 53,915 & 0.86 \\
\hline trust & - & - & - & - & - & - & - & - & - & - & 29,255 & 0.82 \\
\hline ALL & $\mathbf{4 , 5 0 0}$ & $\mathbf{0 . 5 3}$ & $\mathbf{3 , 7 7 7}$ & $\mathbf{0 . 6 7}$ & $\mathbf{2 1 , 0 5 1}$ & $\mathbf{0 . 4 9}$ & $\mathbf{1 , 9 9 1 , 1 8 4}$ & - & $\mathbf{5 2 , 9 2 5}$ & $\mathbf{0 . 7 8}$ & $\mathbf{7 9 0 , 0 5 9}$ & $\mathbf{0 . 8 3}$ \\
\hline
\end{tabular}

Table 6: Comparison (in $F$-score) of our results with GRNNs to published literature. MD = Mohammad (2015). Note: For space restrictions, we take the liberty of using the last name of only the first author of each work.

\begin{tabular}{|l|l|l|l|l|}
\hline Emotion & SGD & MNB & PRCPTN & PAC \\
\hline baseline & 60.00 & 60.00 & 60.00 & 60.00 \\
\hline \hline admiration & 78.30 & 78.01 & 74.24 & 79.86 \\
\hline amazement & 37.57 & 35.71 & 42.51 & 46.69 \\
\hline ecstasy & 51.53 & 51.89 & 47.37 & 53.53 \\
\hline grief & 38.64 & 36.94 & 37.33 & 48.10 \\
\hline loathing & 0.00 & 0.00 & 2.09 & 2.99 \\
\hline rage & 3.47 & 4.49 & 14.02 & 17.04 \\
\hline terror & 33.23 & 44.12 & 40.48 & 47.00 \\
\hline vigilance & 2.53 & 2.56 & 5.52 & 8.42 \\
\hline plutchik-1 & $\mathbf{6 0 . 2 6}$ & $\mathbf{6 0 . 5 4}$ & $\mathbf{5 9 . 1 1}$ & $\mathbf{6 4 . 8 6}$ \\
\hline \hline anger & 19.41 & 13.84 & 24.54 & 29.26 \\
\hline anticipation & 7.46 & 12.63 & 17.29 & 26.70 \\
\hline disgust & 29.51 & 29.87 & 31.83 & 36.60 \\
\hline fear & 21.45 & 25.49 & 30.41 & 33.59 \\
\hline joy & 72.83 & 72.96 & 72.32 & 75.50 \\
\hline sadness & 50.04 & 51.72 & 39.58 & 49.21 \\
\hline surprise & 8.46 & 4.75 & 17.34 & 19.54 \\
\hline trust & 42.09 & 38.52 & 44.48 & 47.51 \\
\hline \hline plutchik-2 & $\mathbf{4 8 . 0 5}$ & $\mathbf{4 8 . 3 3}$ & $\mathbf{4 8 . 6 0}$ & $\mathbf{5 3 . 3 0}$ \\
\hline acceptance & 0.12 & 2.74 & 13.98 & 13.04 \\
\hline annoyance & 80.28 & 80.71 & 78.80 & 81.47 \\
\hline apprehension & 0.80 & 0.00 & 9.72 & 10.66 \\
\hline boredom & 49.53 & 51.27 & 52.02 & 57.84 \\
\hline distraction & 0.00 & 2.99 & 3.42 & 0.00 \\
\hline interest & 21.69 & 30.45 & 34.85 & 44.14 \\
\hline pensiveness & 2.61 & 8.08 & 11.22 & 12.27 \\
\hline serenity & 8.87 & 19.57 & 27.23 & 38.59 \\
\hline plutchik-3 & $\mathbf{6 2 . 2 0}$ & $\mathbf{6 4 . 0 0}$ & $\mathbf{6 4 . 0 4}$ & $\mathbf{6 8 . 1 4}$ \\
\hline \hline ALL & $\mathbf{5 6 . 8 4}$ & $\mathbf{5 7 . 6 2}$ & $\mathbf{5 7 . 2 5}$ & $\mathbf{6 2 . 1 0}$ \\
\hline & & & & \\
\hline
\end{tabular}

Table 3: Results in $F$-score with traditional online classifiers.

We model the set of emotions belonging to each of the 3 circles independently, thus casting each as an 8 -way classification task. Inspired by observations from the literature and our own annotation study, we limit our data to tweets of at least 5 words with an emotional hashtag occurring at the end. We then split the data representing each of the 3 circles into $80 \%$ training (TRAIN), $10 \%$ development (DEV), and $10 \%$ testing (TEST). As mentioned above, we run experiments with a range of online, out-of-core classifiers as well as the
GRNNs. To train the GRNNs, we optimize the hyper-parameters of the network on a development set as we describe below, choosing a vocabulary size of $80 \mathrm{~K}$ words (a vocabulary size we also use for the out-of-core classifiers), a word embedding vector of size 300 dimensions learnt directly from the training data, an input maximum length of 30 words, 7 epochs, and the Adam (Kingma and Ba, 2014) optimizer with a learning rate of 0.001 . We use 3 dense layers each with 1,000 units. We use dropout (Hinton et al., 2012) for regularization, with a dropout rate of 0.5 . For our loss function, we use categorical cross-entropy. We use a minibatch (Cotter et al., 2011) size of 128. We found this architecture to work best with almost all the settings and so we fix it across the board for all experiments with GRNNs.

Results with Traditional Classifiers Results with the online classifiers are presented in terms of $F$-score in Table 3. As the table shows, among this group of classifiers, the Passive Agressive classifier (PAC) acquires the best performance. PAC achieves an overall $F$-score of $64.86 \%$ on plutchik-1, $53.30 \%$ on plutchik-2, and $68.14 \%$ on plutchik-3, two of which are higher than an arbitrary baseline ${ }^{3}$ of $60 \%$.

Results with GRNNs Table 4 presents results with GRNNs, compared with the best results using the traditional classifiers as acquired with PAC. As the table shows, the GRNN models are very successful across all the 3 classification tasks. With GRNNs, we acquire an overall $F$-scores of: $91.21 \%$ on plutchik-1, $82.32 \%$ on plutchik-2, and $87.47 \%$ on plutchik-3. These results are $26.35 \%, 29.02 \%$, and $25.37 \%$ higher than PAC, respectively.

Negative Results We experiment with aug-

\footnotetext{
${ }^{3}$ The arbitrary baseline is higher than the majority class in the training data in any of the 3 cases.
} 


\begin{tabular}{|l|l|l|l|l|}
\hline & PAC & \multicolumn{3}{|c|}{ GRNNs } \\
\hline Emotion & f-score & prec & rec & f-score \\
\hline admiration & 79.86 & 94.53 & 95.28 & 94.91 \\
\hline amazement & 46.69 & 90.44 & 89.02 & 89.73 \\
\hline ecstasy & 53.53 & 83.49 & 90.01 & 86.62 \\
\hline grief & 48.10 & 85.07 & 81.13 & 83.05 \\
\hline loathing & 2.99 & 83.87 & 54.17 & 65.82 \\
\hline rage & 17.04 & 80.00 & 75.11 & 77.48 \\
\hline terror & 47.00 & 91.15 & 84.01 & 87.44 \\
\hline vigilance & 8.42 & 71.93 & 70.69 & 71.30 \\
\hline plutchik-1 & $\mathbf{6 4 . 8 6}$ & $\mathbf{9 1 . 2 6}$ & $\mathbf{9 1 . 2 4}$ & $\mathbf{9 1 . 2 1}$ \\
\hline \hline anger & 29.26 & 74.95 & 69.20 & 71.96 \\
\hline anticipation & 26.70 & 70.05 & 69.00 & 69.52 \\
\hline disgust & 36.60 & 82.18 & 68.84 & 74.92 \\
\hline fear & 33.59 & 73.74 & 72.51 & 73.12 \\
\hline joy & 75.50 & 90.96 & 93.88 & 92.40 \\
\hline sadness & 49.21 & 73.20 & 82.04 & 77.37 \\
\hline surprise & 19.54 & 85.60 & 67.40 & 75.42 \\
\hline trust & 47.51 & 82.43 & 76.83 & 79.53 \\
\hline \hline plutchik-2 & $\mathbf{5 3 . 3 0}$ & $\mathbf{8 2 . 5 3}$ & $\mathbf{8 2 . 4 6}$ & $\mathbf{8 2 . 3 2}$ \\
\hline acceptance & 13.04 & 77.10 & 71.76 & 74.33 \\
\hline annoyance & 81.47 & 91.46 & 95.01 & 93.20 \\
\hline apprehension & 10.66 & 80.40 & 61.07 & 69.41 \\
\hline boredom & 57.84 & 85.95 & 84.40 & 85.16 \\
\hline distraction & 0.00 & 87.50 & 25.00 & 38.89 \\
\hline interest & 44.14 & 86.79 & 78.38 & 82.37 \\
\hline pensiveness & 12.27 & 91.87 & 43.24 & 58.80 \\
\hline serenity & 38.59 & 82.15 & 78.16 & 80.11 \\
\hline plutchik-3 & $\mathbf{6 8 . 1 4}$ & $\mathbf{8 8 . 9 4}$ & $\mathbf{8 9 . 0 8}$ & $\mathbf{8 8 . 8 9}$ \\
\hline ALL & $\mathbf{6 2 . 1 0}$ & $\mathbf{8 7 . 5 8}$ & $\mathbf{8 7 . 5 9}$ & $\mathbf{8 7 . 4 7}$ \\
\hline & & & & \\
\hline
\end{tabular}

Table 4: Results with GRNNs across Plutchik's 24 emotion categories. We compare to bestperforming traditional classifier (i.e. Passive Aggressive).

menting training data reported here in two ways: 1) For each emotion type, we concatenate the training data with training data of tweets that are more (or less) intense from the same sector/dimension in the wheel, and 2) for each emotion type, we add tweets where emotion hashtags occur in the last quarter of a tweet (which were originally filtered out from TRAIN). However, we gain no improvements based on either of these methods, thus reflecting the importance of using high-quality training data and the utility of our strict pipeline.

\subsection{Predicting 8 Primary Dimensions}

We now investigate the task of predicting each of the 8 primary emotion dimensions represented by the sectors of the wheel (where the three degrees of intensity of a given emotion are reduced to a single emotion dimension [e.g., \{ecstasy, joy, serenity $\}$ are reduced to the joy dimension]). We concatenate the $80 \%$ training data (TRAIN) from each of the 3 circles' data into a single training set

\begin{tabular}{|l|l|l|l|}
\hline Dimension & prec & rec & f-score \\
\hline anger & 97.40 & 97.72 & 97.56 \\
\hline anticipation & 91.18 & 89.95 & 90.56 \\
\hline disgust & 96.20 & 93.94 & 95.06 \\
\hline fear & 94.97 & 94.38 & 94.68 \\
\hline joy & 94.61 & 96.40 & 95.50 \\
\hline sadness & 95.52 & 95.25 & 95.39 \\
\hline surprise & 94.99 & 91.62 & 93.27 \\
\hline trust & 96.36 & 97.58 & 96.96 \\
\hline All & $\mathbf{9 5 . 6 8}$ & $\mathbf{9 5 . 6 8}$ & $\mathbf{9 5 . 6 8}$ \\
\hline
\end{tabular}

Table 5: GRNNs results across 8 emotion dimensions. Each dimension represents three different emotions. For example, the joy dimension represents serenity, joy and ecstasy.

\begin{tabular}{|l|l|l|}
\hline Emotion & Volkova (2016) model & This work \\
\hline anger & 12.38 & 74.95 \\
\hline disgust & 5.71 & 82.18 \\
\hline fear & 11.18 & 73.74 \\
\hline joy & 44.57 & 90.96 \\
\hline sadness & 18.04 & 73.20 \\
\hline surprise & 5.33 & 85.60 \\
\hline ALL & $\mathbf{2 6 . 9 5}$ & $\mathbf{8 0 . 1 2}$ \\
\hline
\end{tabular}

Table 7: Comparison (in acc) to (Volkova and Bachrach, 2016)'s model.

(TRAIN-ALL), the $10 \%$ DEV to form DEV-ALL, and the 10\% TEST to form TEST-ALL. We test a number of hyper-parameters on DEV and find the ones we have identified on the fine-grained prediction to work best and so we adopt them as is with the exception of limiting to only 2 epochs. We believe that with a wider exploration of hyperparameters, improvements could be possible. As Table 5 shows, we are able to model the 8 dimensions with an overall superior accuracy of $95.68 \%$. As far as we know, this is the first work on modeling these dimensions.

\section{Comparisons to Other Systems}

We compare our results on the 8 basic emotions to the published literature. As Table 6 shows, on this subset of emotions, our system is $4.53 \%$ (acc) higher than the best published results (Volkova and Bachrach, 2016), facilitated by the fact that we have an order of magnitude more training data. As shown in Table 7, we also apply (Volkova and Bachrach, 2016)'s pre-trained model on our test set of the 6 emotions they predict (which belong to plutchik-2), and acquire an overall accuracy of $26.95 \%$, which is significantly lower than our accuracy. 


\section{Conclusion}

In this paper, we built a large, automatically curated dataset for emotion detection using distant supervision and then used GRNNs to model finegrained emotion, achieving a new state-of-the-art performance. We also extended the classification to 8 primary emotion dimensions situated in psychological theory of emotion.

\section{References}

Conor M. Steckler Aaron C. Weidman and Jessica L. Tracy. 2017. The jingle and jangle of emotion assessment: Imprecise measurement, casual scale usage, and conceptual fuzziness in emotion research. Emotion .

Saima Aman and Stan Szpakowicz. 2007. Identifying expressions of emotion in text. In Text, Speech and Dialogue. Springer, pages 196-205.

Yoshua Bengio, Réjean Ducharme, Pascal Vincent, and Christian Jauvin. 2003. A neural probabilistic language model. Journal of machine learning research 3(Feb):1137-1155.

Yoshua Bengio, Patrice Simard, and Paolo Frasconi. 1994. Learning long-term dependencies with gradient descent is difficult. IEEE transactions on neural networks 5(2):157-166.

Phil Blunsom, Edward Grefenstette, and Nal Kalchbrenner. 2014. A convolutional neural network for modelling sentences. In Proceedings of the 52nd Annual Meeting of the Association for Computational Linguistics. Proceedings of the 52nd Annual Meeting of the Association for Computational Linguistics.

James Bradbury, Stephen Merity, Caiming Xiong, and Richard Socher. 2016. Quasi-recurrent neural networks. arXiv preprint arXiv:1611.01576 .

Kyunghyun Cho, Bart Van Merriënboer, Caglar Gulcehre, Dzmitry Bahdanau, Fethi Bougares, Holger Schwenk, and Yoshua Bengio. 2014. Learning phrase representations using rnn encoder-decoder for statistical machine translation. arXiv preprint arXiv: 1406.1078 .

Junyoung Chung, Caglar Gülçehre, Kyunghyun Cho, and Yoshua Bengio. 2015. Gated feedback recurrent neural networks. In ICML. pages 2067-2075.

Andrew Cotter, Ohad Shamir, Nati Srebro, and Karthik Sridharan. 2011. Better mini-batch algorithms via accelerated gradient methods. In Advances in neural information processing systems. pages 1647-1655.

Munmun De Choudhury, Scott Counts, and Michael Gamon. 2012. Not all moods are created equal! exploring human emotional states in social media.
P. Ekman. 1972. Universal and cultural differences in facial expression of emotion. Nebraska Symposium on Motivation pages 207-283.

Alec Go, Richa Bhayani, and Lei Huang. 2009. Twitter sentiment classification using distant supervision. CS224N Project Report, Stanford 1(12).

Yoav Goldberg. 2016. A primer on neural network models for natural language processing. Journal of Artificial Intelligence Research 57:345-420.

Roberto González-Ibánez, Smaranda Muresan, and Nina Wacholder. 2011. Identifying sarcasm in twitter: a closer look. In Proceedings of the 49th Annual Meeting of the Association for Computational Linguistics: Human Language Technologies: Short Papers-Volume 2. Association for Computational Linguistics, pages 581-586.

Ian Goodfellow, Yoshua Bengio, and Aaron Courville. 2016. Deep learning. MIT Press.

Alex Graves. 2012. Supervised sequence labelling. In Supervised Sequence Labelling with Recurrent Neural Networks, Springer, pages 5-13.

Alex Graves, Abdel-rahman Mohamed, and Geoffrey Hinton. 2013. Speech recognition with deep recurrent neural networks. In Acoustics, speech and signal processing (icassp), 2013 ieee international conference on. IEEE, pages 6645-6649.

Alex Graves and Jürgen Schmidhuber. 2009. Offline handwriting recognition with multidimensional recurrent neural networks. In Advances in neural information processing systems. pages 545-552.

Geoffrey E Hinton, Nitish Srivastava, Alex Krizhevsky, Ilya Sutskever, and Ruslan R Salakhutdinov. 2012. Improving neural networks by preventing coadaptation of feature detectors. arXiv preprint arXiv:1207.0580 .

Sepp Hochreiter and Jürgen Schmidhuber. 1997. Long short-term memory. Neural computation 9(8):1735-1780.

Hyisung C Hwang and David Matsumoto. 2016. Emotional expression. The Expression of Emotion: Philosophical, Psychological and Legal Perspectives page 137.

Ozan Irsoy and Claire Cardie. 2014. Deep recursive neural networks for compositionality in language. In Advances in Neural Information Processing Systems. pages 2096-2104.

Yoon Kim. 2014. Convolutional neural networks for sentence classification. arXiv preprint arXiv: 1408.5882

Diederik Kingma and Jimmy Ba. 2014. Adam: A method for stochastic optimization. arXiv preprint arXiv: 1412.6980 . 
Igor Labutov and Hod Lipson. 2013. Re-embedding words. In $A C L$ (2). pages 489-493.

Quoc V Le and Tomas Mikolov. 2014. Distributed representations of sentences and documents. In ICML. volume 14, pages 1188-1196.

Yann LeCun, Yoshua Bengio, and Geoffrey Hinton. 2015. Deep learning. Nature 521(7553):436-444.

Jiwei Li, Minh-Thang Luong, Dan Jurafsky, and Eudard Hovy. 2015. When are tree structures necessary for deep learning of representations? arXiv preprint arXiv:1503.00185.

Pengfei Liu, Xipeng Qiu, Xinchi Chen, Shiyu Wu, and Xuanjing Huang. 2015. Multi-timescale long shortterm memory neural network for modelling sentences and documents. In EMNLP. Citeseer, pages 2326-2335.

Marco Lui and Timothy Baldwin. 2012. langid. py: An off-the-shelf language identification tool. In Proceedings of the ACL 2012 system demonstrations. Association for Computational Linguistics, pages 25-30.

Andrew L Maas, Raymond E Daly, Peter T Pham, Dan Huang, Andrew Y Ng, and Christopher Potts. 2011. Learning word vectors for sentiment analysis. In Proceedings of the 49th Annual Meeting of the Association for Computational Linguistics: Human Language Technologies-Volume 1. Association for Computational Linguistics, pages 142-150.

Tomas Mikolov, Wen-tau Yih, and Geoffrey Zweig. 2013. Linguistic regularities in continuous space word representations. In Hlt-naacl. volume 13, pages 746-751.

Mike Mintz, Steven Bills, Rion Snow, and Dan Jurafsky. 2009. Distant supervision for relation extraction without labeled data. In Proceedings of the Joint Conference of the 47th Annual Meeting of the ACL and the 4th International Joint Conference on Natural Language Processing of the AFNLP: Volume 2-Volume 2. Association for Computational Linguistics, pages 1003-1011.

Gilad Mishne and Maarten De Rijke. 2006. Capturing global mood levels using blog posts. In $A A A I$ spring symposium: computational approaches to analyzing weblogs. pages 145-152.

Jeff Mitchell and Mirella Lapata. 2010. Composition in distributional models of semantics. Cognitive science 34(8):1388-1429.

Saif M Mohammad. 2012. \#emotional tweets. In Proceedings of the First Joint Conference on Lexical and Computational Semantics-Volume 1: Proceedings of the main conference and the shared task, and Volume 2: Proceedings of the Sixth International Workshop on Semantic Evaluation. Association for Computational Linguistics, pages 246-255.
Saif M Mohammad and Svetlana Kiritchenko. 2015. Using hashtags to capture fine emotion categories from tweets. Computational Intelligence 31(2):301-326.

Thin Nguyen. 2010. Mood patterns and affective lexicon access in weblogs. In Proceedings of the ACL 2010 Student Research Workshop. Association for Computational Linguistics, pages 43-48.

Alexander Pak and Patrick Paroubek. 2010. Twitter as a corpus for sentiment analysis and opinion mining. In $L R E c$. volume 10.

Razvan Pascanu, Tomas Mikolov, and Yoshua Bengio. 2013. On the difficulty of training recurrent neural networks. ICML (3) 28:1310-1318.

Robert Plutchik. 1980. Emotion: A psychoevolutionary synthesis. Harpercollins College Division.

Robert Plutchik. 1985. On emotion: The chickenand-egg problem revisited. Motivation and Emotion 9(2):197-200.

Robert Plutchik. 1994. The psychology and biology of emotion.. HarperCollins College Publishers.

Robert Plutchik. 2001. The nature of emotions human emotions have deep evolutionary roots, a fact that may explain their complexity and provide tools for clinical practice. American scientist 89(4):344-350.

Matthew Purver and Stuart Battersby. 2012. Experimenting with distant supervision for emotion classification. In Proceedings of the 13th Conference of the European Chapter of the Association for Computational Linguistics. Association for Computational Linguistics, pages 482-491.

Jonathon Read. 2005. Using emoticons to reduce dependency in machine learning techniques for sentiment classification. In Proceedings of the ACL student research workshop. Association for Computational Linguistics, pages 43-48.

Yafeng Ren, Yue Zhang, Meishan Zhang, and Donghong Ji. 2016. Context-sensitive twitter sentiment classification using neural network. In $A A A I$. pages 215-221.

Richard Socher, Alex Perelygin, Jean Y Wu, Jason Chuang, Christopher D Manning, Andrew Y Ng, Christopher Potts, et al. 2013. Recursive deep models for semantic compositionality over a sentiment treebank. In Proceedings of the conference on empirical methods in natural language processing (EMNLP). Citeseer, volume 1631, page 1642.

Carlo Strapparava and Rada Mihalcea. 2007. Semeval2007 task 14: Affective text. In Proceedings of the 4th International Workshop on Semantic Evaluations. Association for Computational Linguistics, pages 70-74. 
Kai Sheng Tai, Richard Socher, and Christopher D Manning. 2015. Improved semantic representations from tree-structured long short-term memory networks. arXiv preprint arXiv:1503.00075 .

Yuki Tanaka, Hiroya Takamura, and Manabu Okumura. 2005. Extraction and classification of facemarks. In Proceedings of the 10th international conference on Intelligent user interfaces. ACM, pages 28-34.

Duyu Tang, Bing Qin, and Ting Liu. 2015. Document modeling with gated recurrent neural network for sentiment classification. In Proceedings of the 2015 Conference on Empirical Methods in Natural Language Processing. pages 1422-1432.

Duyu Tang, Furu Wei, Bing Qin, Ming Zhou, and Ting Liu. 2014a. Building large-scale twitter-specific sentiment lexicon: A representation learning approach. In COLING. pages 172-182.

Duyu Tang, Furu Wei, Nan Yang, Ming Zhou, Ting Liu, and Bing Qin. 2014b. Learning sentimentspecific word embedding for twitter sentiment classification. In $A C L$ (1). pages 1555-1565.

Theano Development Team. 2016. Theano: A Python framework for fast computation of mathematical expressions. arXiv e-prints abs/1605.02688. http://arxiv.org/abs/1605.02688.

Svitlana Volkova and Yoram Bachrach. 2016. Inferring perceived demographics from user emotional tone and user-environment emotional contrast. In Proceedings of the 54th Annual Meeting of the Association for Computational Linguistics, ACL.

Wenbo Wang, Lu Chen, Krishnaprasad Thirunarayan, and Amit P Sheth. 2012. Harnessing twitter" big data" for automatic emotion identification. In Privacy, Security, Risk and Trust (PASSAT), 2012 International Conference on and 2012 International Confernece on Social Computing (SocialCom). IEEE, pages 587-592.

Jasy Liew Suet Yan and Howard R Turtle. 2016a. Exploring fine-grained emotion detection in tweets. In Proceedings of NAACL-HLT. pages 73-80.

Jasy Liew Suet Yan and Howard R Turtle. 2016b. Exposing a set of fine-grained emotion categories from tweets. In 25th International Joint Conference on Artificial Intelligence, page 8.

Jasy Liew Suet Yan, Howard R Turtle, and Elizabeth D Liddy. 2016. Emotweet-28: A fine-grained emotion corpus for sentiment analysis .

Changhua Yang, Kevin Hsin-Yih Lin, and Hsin-Hsi Chen. 2007. Emotion classification using web blog corpora. In Web Intelligence, IEEE/WIC/ACM International Conference on. IEEE, pages 275-278.

Meishan Zhang, Yue Zhang, and Duy-Tin Vo. 2016. Gated neural networks for targeted sentiment analysis. In $A A A I$. pages 3087-3093.
Xiang Zhang, Junbo Zhao, and Yann LeCun. 2015. Character-level convolutional networks for text classification. In Advances in neural information processing systems. pages 649-657. 